\title{
Resonances of dynamical checkerboard states in Josephson arrays with self-inductance
}

\author{
M. Barahona, E. Trías, T. P. Orlando, and A. E. Duwel \\ Department of Electrical Engineering and Computer Science, Massachusetts Institute of Technology, Cambridge, Massachusetts 02139
}

H. S. J. van der Zant

Department of Applied Physics, Delft University of Technology, Lorentzweg 1, 2628 CJ Delft, The Netherlands

Shinya Watanabe

CATS, Niels Bohr Institute, Blegdamsvej 17, DK-2100, Copenhagen Ø, Denmark

\author{
S. H. Strogatz \\ Kimball Hall, Theoretical and Applied Mechanics, Cornell University, Ithaca, New York 14853
}

(Received 14 March 1997)

\begin{abstract}
We study the dynamics of fully frustrated, underdamped Josephson arrays. Experiments reveal remarkable similarities among the dc current-voltage characteristics of several kinds of square and triangular arrays, where two resonant voltages are observed. Simulations indicate that a dynamical checkerboard solution underlies these similarities. By assuming such a solution, we reduce the governing equations to three coupled pendulum equations, and thereby calculate the voltages of the intrinsic resonances analytically. [S0163-1829(97)50222-0]
\end{abstract}

Discrete arrays of nonlinear oscillators can exhibit diverse spatiotemporal patterns. Examples include kinks in chains of coupled pendula, neuromuscular waves in the intestine, and modulated waves and chaos in networks of phase-locked loops. ${ }^{1}$ Although such oscillator arrays are difficult to analyze completely, one can often use the symmetries of the system to construct simple patterns composed of spatially repeated "unit cells." Then the governing equations reduce to a much smaller set of equations for each unit cell. This strategy has been used recently to construct rotating spiral waves in a model of discrete excitable media. ${ }^{2}$

In this paper we present experiments, simulations, and analysis on a broad class of discrete arrays of nonlinear Josephson-junction oscillators. Networks ranging from single square and triangular plaquettes to one- and twodimensional arrays are considered. In each case, the junctions are identical and underdamped, and the arrays are driven by a dc bias current. These arrays are known to have complicated dynamics, and only a few analytical results have been obtained. ${ }^{3-6}$ However, one class of arrays is relatively tractable: fully frustrated arrays, i.e., arrays subjected to an applied magnetic field of $f=1 / 2$ flux quanta per plaquette on average. Then the ground state is highly symmetric-the junction phases adopt a checkerboard pattern. ${ }^{7}$ This pattern can be robust: even if the array is driven by an applied dc current, ${ }^{4}$ or a combined ac $+\mathrm{dc}$ current, as in studies of giant Shapiro steps, ${ }^{3,8}$ the checkerboard can persist, though now in the form of a propagating state.

All of the fully frustrated arrays we have tested experimentally exhibit strikingly similar dc current-voltage $(I V)$ characteristics, with two resonant voltages $V_{+}$ $\sim \Phi_{0}\left(L_{s} C\right)^{-1 / 2}$ and $V_{-} \sim \Phi_{0}\left(L_{J} C\right)^{-1 / 2}$. (Here $\Phi_{0}$ is the flux quantum, $L_{s}$ is the loop inductance, $C$ is the junction capacitance, and $L_{J}=\Phi_{0} / 2 \pi I_{c}$ is the Josephson inductance for a junction with a critical current $I_{c}$ ). We show below that these similarities in the $I V$ curves can be explained by assuming that the junction phases are organized into a dynamical checkerboard solution. This ansatz reduces the governing circuit equations to three coupled pendulum equations, which in turn allows us to obtain analytical predictions for the observed resonant voltages.

Previous authors have used the ansatz of a dynamical checkerboard, or some other symmetric pattern, to obtain reduced equations for frustrated Josephson arrays. ${ }^{3-6,9}$ Our analysis extends this work in three respects: (1) Inductance effects are essential to explain some of the experimental re-

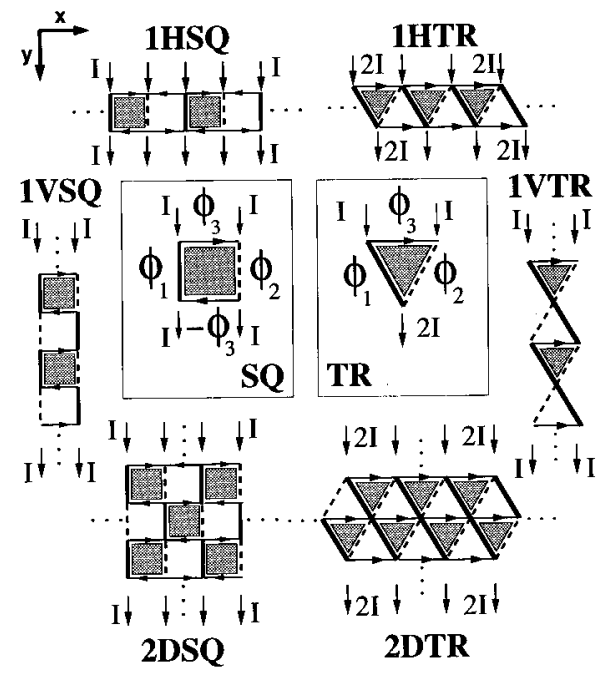

FIG. 1. Arrays and their checkerboard states: Single square (SQ) and single triangular (TR) plaquettes; 1D square (1HSQ and 1VSQ) and 1D triangular (1HTR and 1VTR) arrays; 2D square (2DSQ) and $2 \mathrm{D}$ triangular (2DTR) arrays. The phases of the vertical junctions $\phi_{1}$ and $\phi_{2}$ and the horizontal junction $\phi_{3}$ are defined in the SQ and TR diagrams. The checkerboard solutions are constructed by combining these single plaquettes with their symmetric counterparts. 
sults. Therefore, we include self-inductance in the analysis and simulations as a first approximation. (2) The governing equations for the dynamical checkerboard are shown to have the same algebraic form for all eight arrays in Fig. 1, up to topological factors that encode the array geometry. This shared structure allows a unified analysis of the different networks. (3) Intrinsic resonant voltages are predicted analytically from the reduced equations.

Figure 1 depicts the arrays and their dynamical checkerboard states. Junctions (not shown) exist along each branch, and current is applied at each node along the top and bottom edges. Throughout this paper, "vertical" refers to the direction of current injection. We consider junctions with identical critical currents $I_{c}$, resistances $R_{n}$ and capacitances $C$. Experiments have been performed on arrays of $\mathrm{Nb}-\mathrm{Al}_{2} \mathrm{O}_{x}-\mathrm{Nb}$ junctions ${ }^{10}$ with small damping $\Gamma=\beta_{c}^{-1 / 2}$, where $5<\beta_{c}<25$ is the McCumber parameter. The magnetic field is applied normal to the arrays by an external solenoid. The two-dimensional penetration depth $\lambda=L_{J} / L_{s}$, which can be viewed as a measure of the discreteness of the arrays, ranges from $0.5<\lambda<2$. By use of a diagnostic technique, ${ }^{11}$ we determine the parameters for each array: first, the measurement of $R_{n}$ and $I_{c}(T)$, with $I_{c}(0) R_{n}=1.9 \mathrm{mV}$, yields the temperature-dependent Josephson inductance $L_{J}(T)$; second, from the Fiske modes of the diagnostic we obtain $C, L_{s}$, and the mutual inductance for nearest neighbors, which we find to be small. ${ }^{11,12}$ To facilitate comparisons between different types of arrays, the triangular arrays were built by decimating every other horizontal junction from the corresponding square array; thus their self-inductances are equal.

Figure 2 shows the measured $I V$ curves ( $I$ is the current per vertical junction normalized by $I_{c}$, and $V$ is the voltage per row) for three different geometries at $f=1 / 2$. The signature of all these $I V$ 's is the appearance of jumps at two resonant voltages, $V_{+}$and $V_{-}$. The upper step, which ends at $V_{+}$, is nearly vertical and independent of temperature. For 1HSQ and 2DSQ arrays (the arrays we have studied most

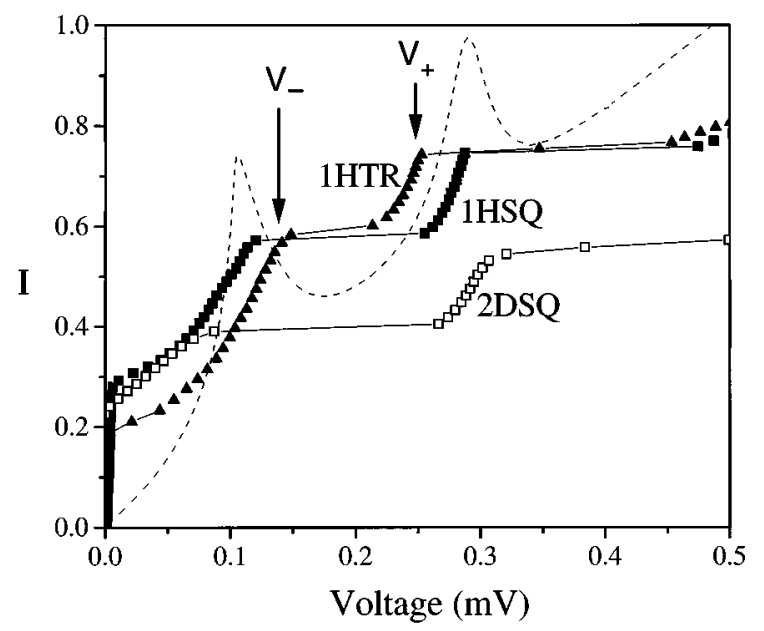

FIG. 2. Experimental $I V$ curves for three arrays: 1HTR array ( $1 \times 9$ plaquettes) with $\beta_{c}=8$ and $\lambda=0.64 ; 1$ HSQ array $(1 \times 7)$ with $\beta_{c}=11$ and $\lambda=0.76$; and 2DSQ array $(7 \times 7)$ with $\beta_{c}=20$ and $\lambda=0.92$. Dashed line, $I V$ from harmonic balance for the $1 \mathrm{HSQ}$ array with the same $\beta_{c}$ and an effective $\lambda_{\text {eff }}=0.61$ which accounts for mutual inductance effects. $V_{+}$and $V_{-}$are indicated for 1HTR. extensively), $V_{+}$is also independent of the number of cells in the $x$ direction. ${ }^{13}$ These results suggest that local geometrical properties, i.e., $L_{s}$, determine the voltage. A temperature-independent voltage $V_{+} \approx \Phi_{0} / \sqrt{L_{s} C}$ is expected from dimensional arguments and is found to be approximately correct experimentally and in simulations. When varying the magnetic frustration, $V_{+}$has the usual periodicity in $f$ and reflection symmetry about $f=1 / 2$ but it is found to be almost independent of $f$ for $0.2<f<0.5$. Thus, although the value of $V_{+}$is similar to the Eck peak in 1D parallel arrays, ${ }^{14}$ it does not have the sine-like $f$ dependence observed in that case. ${ }^{13}$ Moreover, this upper step does not appear for $f<0.2$ or for $\lambda<0.5$ as the system switches directly into row-switched states. The lower voltage $V_{-}$, on the other hand, is temperature dependent, suggesting a dependence on the Josephson inductance; namely, $V_{-} \approx \Phi_{0} / \sqrt{L_{J}(T) C}$, up to a factor of order unity.

The dynamical origin of these two resonances is revealed through numerical simulation of the complete arrays. The governing equations result from current conservation at each node and from fluxoid quantization around each loop. The current $I_{j}$ through each junction, in the resistively and capacitively shunted junction model, is $I_{j}=\sin \phi_{j}+\Gamma \dot{\phi}_{j}+\ddot{\phi}_{j}$ where the currents are in units of $I_{c}$, and time is in units of $\left(L_{J} C\right)^{1 / 2}$, the inverse of the plasma frequency. Fluxoid quantization demands that $\Sigma_{j} \phi_{j}=-2 \pi f-I_{\text {cir }} / \lambda$, where the sum is around a loop, $I_{\text {cir }}$ is the loop current, and only self-(loop)inductances are considered. Simulated $I V$ 's for square arrays are consistent with the experiments. ${ }^{15}$

Furthermore, the simulations at $f=1 / 2$ suggest that solutions with wavelength equal to two plaquettes, as shown in Fig. 1, underlie the observed numerics. These dynamical checkerboard solutions are constituted by single plaquettes (SQ and TR as shown) paired with their symmetric counterparts. Under these symmetry constraints, the number of relevant variables is greatly reduced and the dynamics of the whole array is governed by a set of three coupled equations. Moreover, the equations for each of the eight arrays in Fig. 1 can be recast in the same algebraic form, up to constant factors that encode the array topology. In this unified formulation, the fluxoid quantization condition at $f=1 / 2$ is

$$
\phi_{1}-\phi_{2}-h \phi_{3}=-\pi+I_{3} /\left(\lambda m_{y}\right)
$$

and the two current conservation conditions are

$$
I_{1}+I_{2}=2 I \text { and } I_{2}-I_{1}=2 m_{x} I_{3} / m_{y} .
$$

Here $h$ is the number of horizontal junctions per cell $(h=2$ for square and $h=1$ for triangular arrays), and $m_{y}=1+\nu_{y} / 2$ where $\nu_{y}$ is the number of neighboring cells in the $y$ direction; likewise, $m_{x}=1+\nu_{x} / 2$. For example, the 1HSQ array has $h=2, m_{y}=1$, and $m_{x}=2$, and the 1VTR array has $h=1, m_{y}=2$, and $m_{x}=1$.

It is convenient to introduce three new variables: $\phi=\left(\phi_{1}+\phi_{2}\right) / 2$, the average of the vertical phases; $\theta=\left(\phi_{2}-\phi_{1}-\pi\right) / 2$, which measures how much the difference of the vertical phases differs from $\pi$; and $\varphi=\phi_{3}$, the top horizontal phase. When the arrays have horizontal junctions, i.e. $h \neq 0$, Eqs. (1) and (2) can be rewritten as a system of three coupled nonlinear pendulum equations: 


$$
\begin{gathered}
\ddot{\phi}+\Gamma \dot{\phi}-\sin \theta \sin \phi=I, \\
\ddot{\theta}+\Gamma \dot{\theta}+\cos \phi \cos \theta=-\lambda m_{x}(2 \theta+h \varphi), \\
\ddot{\varphi}+\Gamma \dot{\varphi}+\sin \varphi=-\lambda m_{y}(2 \theta+h \varphi) .
\end{gathered}
$$

Numerical simulations indicate that, for the parameter regimes considered, $\phi$ is approximately a uniformly whirling phase with frequency $\omega$, whereas both $\theta$ and $\varphi$ librate sinusoidally at $\omega$. Thus, we approximate the solution as $\phi=\omega t+k, \theta=B \sin (\omega t+\alpha)$, and $\varphi=A \sin \omega t$, where $\alpha, k$, $A, B$, and $\omega$ are constants to be determined for each set of driving conditions $\{I, \Gamma, \lambda\}$. A harmonic balance calculation with this assumed solution yields an $I V$ characteristic with two resonant peaks, as shown by the dashed line in Fig. 2 for the 1 HSQ device. These peaks are typically associated with a loss of stability of the underlying dynamical state, thus explaining the experimental steps. This semi-analytic $I V$ approximates the voltages of the resonant steps reasonably well, although it does not provide a quantitative fit to the measured currents. The details of this calculation will be given elsewhere. $^{15}$

Approximate formulas for the resonant voltages can be obtained via a further simplification. Since the libration amplitudes $A$ and $B$ are observed to be small for a wide range of bias current, we regard both $\varphi$ and $\theta$ as small oscillations driven by the whirling mode $\phi$. Then Eqs. (4) and (5) can be linearized and, in the limit of small damping $(\Gamma \ll 1)$, these two equations have two resonance frequencies at

$$
\omega_{ \pm}^{2}=1 / 2+\lambda m_{x} \sigma \pm\left[\left(1 / 2+\lambda m_{x} \sigma\right)^{2}-2 \lambda m_{x}\right]^{1 / 2},
$$

where $\sigma=1+h m_{y} /\left(2 m_{x}\right)$. These frequencies agree with the location of the peaks of the $I V$ from the full harmonic balance calculation. To clarify the physical meaning of the two resonances $\omega_{ \pm}$, consider the limit of small inductance, $\lambda \gg 1$. Then, $\omega_{+} \approx \sqrt{2 \lambda m_{x} \sigma}=b_{+} \sqrt{\lambda}$ and $\omega_{-}$ $\approx \sigma^{-1 / 2}=b_{-}$. The corresponding voltages are $V_{+} \approx b_{+} \Phi_{0} /$ $\left(2 \pi \sqrt{L_{s} C}\right)$ and $V_{-} \approx b_{-} \Phi_{0} /\left[2 \pi \sqrt{L_{J}(T) C}\right]$, as guessed earlier from dimensional arguments. Thus, $V_{+}$is temperature independent while $V_{-}$depends on $T$; and $b_{+}$and $b_{-}$are combinations of topological factors of order unity. [For the single triangle (TR), $b_{+}=\sqrt{3}$, as suggested by Yukon and Lin. $\left.{ }^{9}\right]$

Figure 3 shows good agreement between the temperature dependence of the experimental (squares and triangles) and predicted (lines) normalized voltages $V_{+} / b_{+}$and $V_{-} / b_{-}$for 1HSQ, 2DSQ, and 1HTR arrays. The fact that Eq. (6) (dashed line) consistently overestimates the value for $V_{+} / b_{+}$can be attributed to an underestimation of the inductance since simulations with self-inductance alone (pluses in the figure) agree well with the theory. When simulations with the full inductance matrix are performed (crosses), the resonant voltage moves down toward the experimental data. The main effect of the mutual inductive coupling can be approximated by an effective inductance $L_{s, \text { eff }}=L_{s}\left\{1+M\left(\nu_{x}+\nu_{y}\right)\right\}$ where $M \approx 0.12$ is the ratio of the magnitude of the nearest-neighbor inductance to the selfinductance in square arrays. ${ }^{12}$ The solid line corresponds to $V_{+} / b_{+}$calculated from Eq. (6) with this effective inductance and predicts well both the full inductance simulations
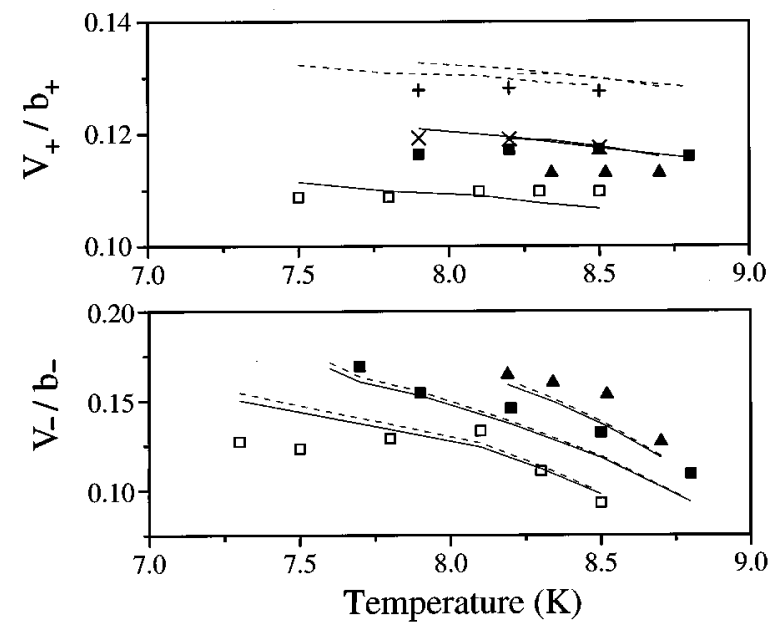

FIG. 3. Normalized resonant voltages vs $T$, measured for the arrays presented in Fig. 2: 1HTR (solid triangles), 1HSQ (solid squares), and 2DSQ (open squares). Dashed lines, Eq. (6). Solid lines, Eq. (6) with $L_{s, \text { eff }}$ as defined in the text. Values of $V_{+} / b_{+}$ from numerical simulations of the 1HSQ array with only selfinductance (pluses) and the full inductance matrix (crosses) are also shown. The 1HTR array was built from a 1HSQ by decimation of every other horizontal junction. Therefore, the geometrical unit cells are identical and $L_{s}=23 \mathrm{pH}$ and $C=300 \mathrm{fF}$ for the three arrays.

and the experimental data. In addition, the values and temperature dependence of $V_{-} / b_{-}$agree well with the theory and simulations and, as expected, no inductance effects are visible.

Both the horizontal junctions and the inductance play essential roles in the phenomena described above; without them, one or both of the observed resonances would be lost. To see this, consider three well-studied limiting cases. First, if the horizontal junctions are absent, the SQ and TR arrays are simply two-junction superconducting quantum interference devices with inductances, and 1HSQ and 1HTR become 1D parallel arrays, which can be regarded as discrete versions of long Josephson junctions. The dynamics of these systems are then governed by only two equations, Eqs. (3) and (4) with $h=0$, since $\varphi$ is no longer a valid dynamical variable. This system has a single linear resonance, at a frequency $\omega=\sqrt{2 \lambda m_{x}}=\left.\omega_{+}\right|_{h=0}$. Second, in the limit $\lambda \rightarrow \infty$ where inductances are neglected, there is no divergence in the system (3)-(5) since $\theta \equiv-h \varphi / 2$. Thus, Eqs. (4) and (5) are combined to eliminate $\lambda(2 \theta+h \varphi)$ and the dynamics of the system is then governed by only two equations: Eq. (3) and a reduced equation for $\theta$,

$$
\ddot{\theta}+\Gamma \dot{\theta}+\frac{1}{\sigma} \frac{h}{2}\left\{\sin \left(\frac{2}{h} \theta\right)+\frac{m_{y}}{m_{x}} \cos \theta \cos \phi\right\}=0 .
$$

(These equations include as a special case the 2DSQ system previously studied in Refs. 3 and 4 Note also that when $\lambda \rightarrow \infty$, the SQ and 2DSQ equations are identical but this is no longer true when inductances are taken into account.) In conclusion, in the absence of inductance there is a single linear resonant frequency $\omega=\sigma^{-1 / 2}=\left.\omega_{-}\right|_{\lambda \rightarrow \infty}$. Third, if there are neither horizontal junctions nor inductances, the sole dynamical Eq. (3) has no resonances. 
These limiting cases also suggest a heuristic explanation for the origin of the resonances. At $V_{-}$, as the checkerboard slides across the array, it strongly excites spin waves, or equivalently, "ringing', oscillations of the junctions at an eigenfrequency close to their characteristic plasma frequency $\left[\left(L_{J}(T) C\right]^{-1 / 2}\right.$. This resonance requires horizontal junctions but not inductance, and is temperature dependent. In contrast, the resonance at $V_{+}$is due to excitation of electromagnetic modes of the array. This resonance requires inductance but not horizontal junctions, and depends only on local geometrical properties of the array that are temperature independent. The corresponding eigenmode is mainly related to oscillations in the induced flux per cell.

The role of the horizontal junctions can be further explored by considering an anisotropic network where horizontal and vertical junctions are fabricated with the same process and differ only in their area. Then the ratio of their critical currents, $\eta=I_{c 1} / I_{c 3}$, parametrizes the anisotropy of the array. In this case, the results from Eqs. (3)-(6) are still valid with a renormalized $m_{y}^{\prime}=m_{y} \eta$, which specifies the participation of the horizontal junctions in the dynamics of the array.

Finally, we emphasize that our analysis is based on the assumption of a dynamical checkerboard state. Unfortunately, very little is known about the conditions for its global stability. If it is unstable, or if the array organizes itself in some alternative stable state, the dynamics are not yet understood. For instance, simulations of 2DTR arrays with low $\beta_{c}$ seem to show a different solution (the "ribbon state") where the horizontal junctions are essentially inactive and a checkerboard is formed by double cells. ${ }^{9}$ This solution is analogous to the striped columnar dynamical configuration observed in $f=1 / 2$ Shapiro steps in square arrays. ${ }^{16}$ In both cases, the arrays effectively behave as a collection of inphase rows and, thus, have only one resonant voltage at $\left.V_{+}\right|_{h=0}$. Experiments on triangular arrays will address these issues separately. ${ }^{17}$ Also, if the junctions are highly underdamped, the checkerboard state in 2DSQ arrays can slide chaotically. ${ }^{4}$ The conditions for the stability and the temporal periodicity of the checkerboard state, and the dynamics associated with other possible states, are challenging problems for future investigation.

We thank P. Caputo, S. Yukon, N. Lin, and A. Ustinov for useful discussions and for sharing their unpublished results on triangular arrays. This research was supported in part by NSF Grant No. DMR-9402020 and Grant No. DMS9500948 and by Rome Laboratory (AFMC) Grant No. F 30602-96-2-0059. A.E.D. and E.T. acknowledge partial support from the NSF.
${ }^{1}$ A.T. Winfree, The Geometry of Biological Time (Springer, New York, 1980); V.S. Afraimovich et al., Stability, Structures, and Chaos in Nonlinear Synchronization Networks (World Scientific, Singapore, 1994).

${ }^{2}$ J.E. Paullet and G.B. Ermentrout, SIAM J. Appl. Math. 54, 1720 (1994).

${ }^{3}$ M.S. Rzchowski, L.L. Sohn, and M. Tinkham, Phys. Rev. B 43, 8682 (1991); L.L. Sohn et al., ibid. 45, 3003 (1992); L.L. Sohn and M. Octavio, ibid. 49, 9236 (1994).

${ }^{4}$ M. Octavio, C. B. Whan, U. Geigenmüller, and C. J. Lobb, Phys. Rev. B. 47, 1141 (1993).

${ }^{5}$ R. Bhagavatula, C. Ebner, and C. Jayaprakash, Phys. Rev. B 45, 4774 (1992); J. Kim et al., ibid. 49, 459 (1994); I.F. Marino and T.C. Halsey, ibid. 50, 6289 (1994).

${ }^{6}$ A.S. Landsberg, Y. Braiman, and K. Wiesenfeld, Phys. Rev. B 52, 15458 (1995).

${ }^{7}$ S. Teitel and C. Jayaprakash, Phys. Rev. Lett. 51, 1999 (1983); S. Teitel and C. Jayaprakash, Phys. Rev. B 27, 598 (1983).
${ }^{8}$ K.H. Lee, D. Stroud, and J.S. Chung, Phys. Rev. Lett. 64, 962 (1990).

${ }^{9}$ S.P. Yukon and N.C.H. Lin, in Macroscopic Quantum Phenomena and Coherence in Superconducting Networks, edited by C. Giovannella and M. Tinkham (World Scientific, Singapore, 1995), p. 351; IEEE Trans. Appl. Supercond. 5, 2959 (1995).

${ }^{10}$ HYPRES Inc., Elmsford, NY 10523.

${ }^{11}$ H.S.J. van der Zant, R.A.M. Receveur, T.P. Orlando, and A.W. Kleinsasser, Appl. Phys. Lett. 65, 2102 (1994).

${ }^{12}$ R.D. Bock, J.R. Phillips, H.S.J. van der Zant, and T.P. Orlando, Phys. Rev. B 49, 10009 (1994).

${ }^{13}$ E. Trías, M.S. thesis, MIT, 1995.

${ }^{14}$ H.S.J. van der Zant and T.P. Orlando, J. Appl. Phys. 76, 7606 (1994).

${ }^{15}$ E. Trías et al. (unpublished).

${ }^{16}$ D. Domínguez and J.V. José, Phys. Rev. Lett. 69, 514 (1992).

${ }^{17}$ A.E. Duwel et al. (unpublished). 